\title{
Viewpoint: New Approaches and Protocols for Grazing Management Research
}

\section{By Mort Kothmann, Richard Teague, Heriberto Díaz-Solís, and William Grant}

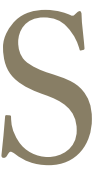
toddart and Smith ${ }^{1}$ defined range management as "...the science and art of planning and directing range use...” (p. 2). Over the past 100 years, the art and science of grazing management have sometimes taken divergent paths. Early observations that moderate stocking rates improved rangeland health and vegetation composition were followed by research that identified the ecological and physiological mechanisms for vegetation responses to grazing. However, these scientific principles have seen limited application in grazing systems research. Briske et al. ${ }^{2}$ concluded, "The ecological relationships of grazing systems have been reasonably well resolved, at the scales investigated, and a continuation of costly grazing experiments adhering to conventional research protocols will yield little additional information." If further research "at the scales investigated" and "adhering to conventional research protocols" is not warranted, does this mean there should be no further grazing management research?

We suggest that more grazing management research is needed, but with new protocols. Heady ${ }^{3}$ stated, "The time has arrived to organize the information from research or few-factor analysis with whole-system practical experience so that specialized grazing systems will be even better" (p. 897). We suggest that achieving this laudable goal will require addressing grazing management research not as the comparison of fixed treatments with the "winner" recommended as a best management practice, but as an analysis of a dynamic system that requires continuous management. In this paper, we present modeling as one component of a new research protocol for grazing management research. Although researchers have modeled ecosystem functions for over 40 years, there are few applications focused specifically on grazing management.

\section{Observations on the Relationship between Science and Management}

Forester, ${ }^{4}$ considered to be the father of systems analysis, addressed the respective roles of both the art and science of management and, although his discipline was business management, his observations are relevant to rangeland management.

The art develops through empirical experience but in time ceases to grow because of the disorganized state of its knowledge. When the need and necessary foundations coincide, a science develops to explain, organize, and distill experience into a more compact and usable form. As the science grows, it provides a new basis for further extension of the art...

The manager has often found that management science did not deal with his most urgent problems. It has not learned how to take into account the variables that he knows to be important. It is not cast in a language with which he is familiar...

Currently, few grazing management research studies are being conducted on US rangelands. However, innovative ranch managers are using adaptive management techniques to successfully develop more complex, site-based, grazing management programs on their ranches. ${ }^{5}$ Successful ranch managers apply adaptive management principles because what is optimal for one ranch may not be optimal for another ranch. Past grazing management research has not evaluated this adaptive management approach. Research should identify key processes and principles and should structure them in management-oriented decision-support models that allow managers to develop unique grazing management programs that can successfully adapt to everchanging combinations of physical, biotic, and social resources and needs.

Provenza ${ }^{6}$ defined adaptive management as the continuing process of defining visions and goals $\rightarrow$ setting objectives $\rightarrow$ implementing practices $\rightarrow$ monitoring $\rightarrow$ changing behavior in repeated learning cycles. Adaptive management 
is not looking for prescriptions but rather understandable descriptions of the components, mechanisms, and processes that can be used in repeated cycles of adaptive management to develop successful grazing management programs. This view of management does not ask science to be a predictive oracle attempting to guide grazing management. Rather, science is a way to understand the processes of nature and to monitor and assess ecosystem responses to management. Adaptive management is about flexibility in the face of change and uncertainty.

Management implies making decisions, which involves flexibility. Adaptive management involves continuously applying the cycle described by Provenza. ${ }^{6}$ Grazing management on rangelands involves various seasons of use, kinds and classes of animals, kinds and condition of vegetation, characteristics of the soils, topography, and climate, levels of management knowledge and skill, and economic and financial resources that are unique to each ranch. This complexity greatly exceeds the ability of traditional field research to test the vast array of possible "grazing systems." According to Forester, researchers should identify the essential components, interrelationships, and processes of successful management programs in order to understand how leading practitioners manage successful programs. It is the role of researchers to formulate and quantify the concepts and principles so that other managers using adaptive management on their ranch can successfully apply the principles, not a best-management practice.

\section{Changing the Research Protocol}

Field experiments using traditional analysis of variance (ANOVA) statistical designs compare responses (e.g., plant and animal production) of defined treatments using the null hypothesis (no difference) as the default. If the null hypothesis is rejected, then "winners" and "losers" are declared. True replication is usually scant or absent. In the comparison of management "systems," specific quantitative cause and effect hypotheses are generally absent. Because treatments are fixed, management during the course of the study is constant. This eliminates the possibility of using adaptive management to capitalize on emergent opportunities or problems. Regression equations can be fit to data sets, but they have limited portability to new locations and very limited capability for formulating new management options.

We propose that grazing research of the future should formulate quantitative functions relating plant and animal responses to system components that are structured into simulation models. These models become the default hypotheses tested using field data from research studies or from observations on managed ranches. ${ }^{7}$ This approach allows the evaluation of whole managed systems, not just fixed treatments. As the model is tested repeatedly against different data sets, it is either validated or specific components are rejected and then modified to incorporate the new information. Such models embody much information about the components and their functional relationships in managed ecosystems. Simulation models constructed based on functional relationships can be used regionally and can provide the opportunity to explore many management alternatives. Again, promising new management options identified from the simulation experiments will need to be tested with field studies. This iterative approach of using field data to formulate models, applying the models to field problems, modifying the models, and testing them with new field data was described by Walters. ${ }^{8}$

Most ecosystem models constructed over the past 40 years have been complex mechanistic models. Some have been proposed as decision tools (e.g., Simulation of Production and Utilization of Rangelands [SPUR]) for management of grazing lands, although their use by managers has been very limited. The explicit, detailed representation of ecological processes in these models is scientifically interesting, but the complexity of the databases required to parameterize them and the complexity of their output limits their application in management of grazing lands. The proposed use of increasingly detailed mechanistic models as decision-making tools for management of grazing lands has drawn attention to key problems inherent in the more detailed approach. For example, the number of parameters grows exponentially as processes are decomposed into finer grain and lower levels of organization; small errors in the estimation of individual parameters may have large cumulative effects on model performance; and predictions from the detailed model still must be tested by reference to, and experiments conducted at, the higher levels of aggregation in which managers operate. Thus, while detailed mechanistic simulation models are attractive to researchers for their predictive power, they find few applications in management settings.

An effective model that supports decision making should be suitable for use by managers. The manager must be able to parameterize the model using information that is readily available, and the output must be understandable and applicable to management decisions, i.e., it must not require a skilled research scientist to use it. The parameters need to be observable and measureable in a field setting. These models must be flexible, allowing managers to make adjustments based on monitoring data (e.g., adaptive management). We have found that models with parameters based at the management level of organization are more stable and useful for management than the complex mechanistic models. Management itself should be viewed as experimentation at the highly aggregated level of the intact system. ${ }^{8}$ This concept has not been widely accepted or used in the modeling community.

Why do we recommend modeling as a "new" research approach for grazing management when there have been so many models built? This is a reasonable question. Modeling requires conceptualization of the system with specification 
of the components, structure, and processes at specific levels of organization. It requires quantification of interdependencies of system components. The model becomes a dynamic complex hypothesis of system functions and relationships. Inputs and outputs of the model can be tested against field data. There are many ways to represent the biotic, physical, and social components of grazed systems. Most modeling research has been conducted by scientists who have been trained in reductionist approaches, and they have built mechanistic models that are based on lower levels of system organization. Our approach has been to conceptualize model components at the management level of organization. This greatly reduces the number of model components and functions and provides greater model stability. It is not the purpose of this paper to describe this process, but references are provided that will allow the interested reader to access publications reporting this research.

We have developed and evaluated simple models that represent selected ecological dynamics of grazing-land systems, and these can be parameterized based on information that is readily available. These models support specific management decisions with output that is both understandable and useful to managers. Philosophically, our modeling approach is to develop the simplest model that will represent the key state variables and processes of interest of the system and test them against field data to determine whether additional components are needed for the model. Components that are not needed for the model to adequately represent the system processes are also removed, much like selecting independent variables that need to be included in the model when using the stepwise regression technique.

Two models that we have designed to support adaptive grazing management are presented as examples of different types of management models. Simple Ecological Sustainability Simulator (SESS) is a strategic-level model designed to simulate vegetation and livestock responses to management alternatives over decades. The Grazing Manager (TGM) is an operational-level decision-support model that facilitates planning, monitoring, and analysis of grazing management based on annual forage balance (e.g., forage production and forage demand). These models are not new approaches to grazing management, but they are examples of models designed specifically to support management decisions. We think that the concepts and approaches used to develop them can provide some new insight for the development of a broader suite of management-oriented models.

\section{SESS: A Simple Ecological Sustainability Simulator}

We developed, parameterized, and corroborated SESS as a rangeland management model for Texas and northern Mexico. ${ }^{9-14}$ It is capable of long-term (20-50-year) simulations of vegetation responses, animal performance, and economic consequences of different ranch grazing-management options. This model represents a "first step" in quantifying ecological processes and integrating them into an adaptive management framework.

SESS was developed based on simple relationships and expertise of range management researchers of north Mexico and south Texas. The idea of large-scale experiments is new. SESS was built from our years of research experience combined with our knowledge of ranching enterprises and the literature. This year Díaz-Solís is starting with this approach in 10 ranches in different ecosystems of Coahuila, Mexico. This, coupled with current on-ranch research, allows assessment of the short- and long-term biological and economic implications of different range management options. Díaz-Solís has used the original SESS model as a decision-making aid among groups of ranchers in northern Mexico for 5 years.

Models have great potential to enhance our knowledge of ecosystem function and to serve natural resource managers as decision-making aids. Model simulations can address issues at the whole-farm or ranch level, and they can be used to assess the effects of different physical properties, input levels, weather sequences, and management actions in a variety of land management units. We have used the SESS rangeland simulation model to examine the consequences of using different grazing management under a wide range of physical conditions, weather sequences, and management options. This is not possible using field experiments alone. This approach allows us to assess the potential of different management practices to achieve economic and ecosystem service goals in these southern Great Plains agroecosystems.

The process of developing a quantitative model highlights fuzzy thinking about the system and identifies knowledge gaps and limitations. During the development of SESS, we found that the forage harvestability threshold varied with the productivity of the site (e.g., forage on low-productivity sites had greater availability at lower levels of standing crop than on high-productivity sites). The National Research Council (NRC) ${ }^{15}$ presented an equation for adjusting animal forage intake as a function of forage standing crop. Using the NRC equation for adjusting intake as a function of forage standing crop, SESS predicted that livestock husbandry was not feasible in sites with precipitation lower than $400 \mathrm{~mm}$ per year because of severely reduced intake. This led us to develop an alternative hypothesis (e.g., harvest efficiency of herbivores as a function of standing crop is higher in less productive sites). This is an example of how the development and use of models can identify faulty relationships in current science and can suggest alternative hypotheses. Developing more robust and widely applicable functions for grazing management processes is a valid role for traditional science.

Continued use of SESS over a period of years for a variety of different sites and management programs will identify 
existing components and processes that should be modified or new variables that should be included to provide more robust predictions. An iterative process consisting of prediction with the model, collection of field data, and adjustment of model structure and parameters will lead to a more robust model that can serve as a general decision support tool to improve decision making of land managers and policy makers. ${ }^{8}$

\section{The Grazing Manager (TGM): An Operational- Level Grazing Management Model}

Scientists and managers agree that stocking rate is the most critical grazing management variable. However, the inability of managers to adequately monitor and project annual forage production and availability and to relate forage values to animal production and carrying capacity on their ranch has been a major management limitation. Traditional grazing studies provide average stocking rate recommendations, but the manager must then determine when and how much to adjust stocking rates based on variations in forage production. Research has not provided quantitative decision tools to assist managers with these important decisions. TGM is a simulation model for annual forage balance (e.g., supply relative to demand) that was designed as a decision aid for managers to use to plan, monitor, and evaluate grazing on their ranch in an adaptive management context. TGM was designed to capture the basic elements of the "intuitive" management approach used by many successful grazing managers and to convert it to a quantitative dynamic forage balance model, thus making the decisionmaking process explicit. It facilitates quantitative analysis and evaluation of grazing management proposed by the manager (e.g., inputs to the model) and validates results by field monitoring on the ranch. TGM has completed five cycles of development. ${ }^{16-20}$ In each stage of the development process, we have conducted extensive field testing on many ranches.

\section{TGM Design}

TGM was designed as a general model that can be used in many different forage/grazing systems. The following general criteria guided model development.

1. TGM should begin with the simplest model of the forage-balance system that can be conceived. Additional variables should be added only when the model is not adequate to explain observed responses. We eliminated or aggregated variables whenever possible. In short, we kept the model simple and understandable by a manager.

2. TGM should not be site specific, i.e., key model inputs should be entered by the local user. The model should contain the structure for simulation and analysis of input data but should not include site-specific parameters.

3. TGM operates on a daily time step and at the same level of organization as the management decisions it supports (e.g., pastures and herds).
4. TGM variables should be aggregated to use the minimum number of variables possible to address the desired decision analysis. Variable selection is extremely important to making the model both responsive and useable. Explicit representation of detailed components was avoided when an aggregated variable would suffice.

5. All TGM model inputs should be readily available to the manager at reasonable cost of time, labor, and expertise. Keeping the resolution of the model at the management level and selecting aggregated variables facilitate this criterion.

6. TGM should provide useful management information but should not make management recommendations. The manager makes all decisions using analysis that TGM provides.

7. Because management goals and objectives are highly variable, management assumptions should not be made within the model. Our approach was to gather basic inventory and planning data from the manager, to quantitatively analyze the data, to provide a framework for frequent monitoring and updating of plans, and to provide a procedure for on-site validation of the data entered by the manager. This information allows a manager to make better grazing-management decisions.

8. TGM model analyses and reports provide a simple method for the on-site manager to validate the management data they enter against actual system performance monitored in the field.

9. TGM can develop and store annual records of pasture productivity, herd inventory, grazing plans, seasonal forage growth rates, prescribed burns, hay production, and pasture utilization.

10. TGM can be used with grazing methods ranging from continuous stocking to daily rotations.

11. TGM is designed to facilitate and reinforce application of the adaptive management cycle.

TGM software has been evaluated and used on ranches across Texas and in nearly all western states. It can be downloaded free of charge at http://www.thegrazingmanager. com/drupal/.

\section{Future Research Needs Related to Scale}

Field research, faced with significant limitations of resources, has not dealt adequately with the effects of scale, of either space or time. Field research and experience gained by ranchers from commercial ranch operations have identified some broad principles; however, this information base is incomplete. Simulation models are needed to address these effects. However, such models will be frivolous unless model output is corroborated with evidence from commercial ranches and/or field experiments.

Spatial distribution of grazing on rangelands is affected by vegetation composition, stock density, pasture size and 
shape, topography, location of water, and various other factors. Thus, results of scientific grazing experiments conducted with few animals within small areas often contradict the common sense of experienced ranchers. Development of models that simulate the responses of many individual animals grazing within real landscapes is possible with recent increases in the computing speed of personal computers. Such models provide exciting possibilities for identifying and integrating quantitative "local rules" of grazing obtained from grazing experiments with the general, qualitative "landscape rules" of grazing known by ranch managers. Ranchers whose experience has given them a keen eye for recognizing such patterns can evaluate the grazing patterns produced on simulated landscapes.

\section{Summary}

Managers need information to understand functional processes and principles, not prescriptive practices. New approaches for grazing management research must be holistic, straightforward, adaptive, and interactive. Research must be holistic in the sense of recognizing that grazing management is understandable only in relation to the whole ranch enterprise. This is a straightforward procedure if investigations focus on relevant processes at appropriate spatial and temporal scales. It is adaptive and interactive in the sense of viewing grazing management itself as a series of ranch-scale experiments that actively involve ranchers in research design and evaluation. A defining characteristic of such approaches will be the quantitative testing, first via simulation and then in the field, testing specific hypotheses integrated at the whole-ranch scale.

\section{Acknowledgments}

The authors wish to thank several graduate students who have participated in our research programs and several anonymous reviewers of this manuscript whose insightful comments contributed significantly to improving the final product.

\section{References}

1. Stoddart, L. A., And A. D. Smith. 1943. Range management. New York, NY, USA: McGraw-Hill Book Company, Inc. 547 p.

2. Briske, D. D., J. D. Derner, J. R. Brown, S. D. Fuhlendorf, W. R. Teague, K. M. Havstad, R. L. Gillen, A. J. Ash, AND W. D. Willms. 2008. Rotational grazing on rangelands: reconciliation of perception and experimental evidence. Rangeland Ecology and Management 61:3-17.

3. Heady, H. F. 1984. Concepts and principles underlying grazing systems. In: Developing strategies for rangeland management. Boulder, CO, USA: National Research Council, National Academy of Sciences, West View Press. 2022 p.

4. Forester, J. W. 1961. Industrial dynamics. Cambridge, MA, USA: The M.I.T. Press. 464 p.

5. Howell, J. 2008. For the love of land: global case studies of grazing in nature's image. Charleston, SC, USA: BookSurge Publishing. 496 p.
6. Provenza, F. D. 2008. What does it mean to be locally adapted and who cares anyway? Journal of Animal Science 86: E271-E284.

7. Kothmann, M. M., And G. M. Smith. 1983. Evaluating management alternatives with a beef production systems model. Journal of Range Management 36(6):733-740.

8. Walters, C. 1986. Adaptive management of renewable resources. New York, NY, USA: MacMillan. 374 p.

9. Díaz-Solís, H., M. M. Kothmann, W. T. Hamilton, and W. E. Grant. 2003. A simple ecological sustainability simulator (SESS) for stocking rate management on semi-arid grazinglands. Agricultural Systems 76:655-680. Available at: http://dx.doi.org/10.1016/S0308-521X(01)00115-9.

10. Díaz-Solís, H., M. M. Kothmann, W. E. Grant, and R. De Luna-Villarreal. 2006. Application of a simple ecological sustainability simulator (SESS) as a management tool in the semi-arid rangelands of northeastern Mexico. Agricultural Systems 88:514-527. Available at: http://dx.doi.org/10.1016/j. agsy.2005.07.008.

11. Díaz-Solís, H., W. E. Grant, M. M. Kothmann, W. R. Teague, and J. A. Díaz-García. 2009. Adaptive management of stocking rates to reduce effects of drought on cow-calf production systems in semi-arid rangelands. Agricultural Systems 100:43-50. Available at: http://dx.doi.org/10.1016/j. agsy.2008.12.007.

12. Dube, S. 2005. A model for adaptive livestock management on semi-arid rangelands in Texas [dissertation]. College Station, TX, USA: Texas A\&M University. 125 p.

13. Teague, W. R., W. E. Grant, U. P. Kreuter, H. DíazSolís, S. Dube, M. M. Котhmann, W. E. Pinchak, and R. J. Ansley. 2008. An ecological economic simulation model for assessing fire and grazing management effects on mesquite rangelands in Texas. Ecological Economics 64:612-625. Available at: http://dx.doi.org/10.1016/j.ecolecon.2007.04.010.

14. Teague, W. R., W. E. Grant, H. Díaz-Solís, U. P. Kreuter, and M. M. Kothmann. 2009. Economic implications of maintaining rangeland ecosystem health in a semi-arid savanna. Ecological Economics 68(5):1417-1429. Available at: http://dx.doi.org/10.1016/j.ecolecon.2008.10.014.

15. National Research Council. 2000. Nutrient requirements of beef cattle. Seventh revised edition: update 2000. The National Academy of Sciences. Available at: http//:www.nap. edu/openbook/0309069343/html/R11.html. Accessed October 2009.

16. Casco, J. 1987. Stocking rate decision aid [dissertation]. College Station, TX, USA: Texas A\&M University. 226 p.

17. Kothmann, M. M., and R. T. Hinnant. 1990. Grazing management stock adjustments templates. College Station, TX, USA: Texas Agriculture Experiment Station-TAEX, CBZ. 119 p.

18. Kothmann, M. M., and R. T. Hinnant. 1994. The grazing manager version 1 and grazing management stock adjustment templates version 2 [computer program]. College Station, TX, USA: Texas Agricultural Experiment Station MP 1760, Computer Software Documentation Series.

19. Kothmann, M. M., and R. T. Hinnant. 1999. The grazing manager for Windows 95, 98, NT [computer program]. College Station, TX, USA: Department of Rangeland Ecology and Management, Texas Agricultural Experiment Station. 
20. Agren, Inc. 2007. The Grazing Manager software. Available at: http://www.thegrazingmanager.com/drupal/. Accessed October 2009.

Authors are Professor, Dept of Ecosystem Science and Management, Texas AE'M University, College Station, TX 77843,
USA,m-kothmann@tamu.edu (Kothmann); Associate Resident Director and Professor, Texas AgriLife Research and Extension Center (Teague), and Professor, Department of Wildlife and Fisheries Sciences (Grant), Texas AE'M University, College Station, TX 77843, USA; and Investigador Nacional Nivel I, Departamento de Recursos Naturales Renovables, Universidad Autonoma Agraria Antonio Narro (Diaz-Solis).

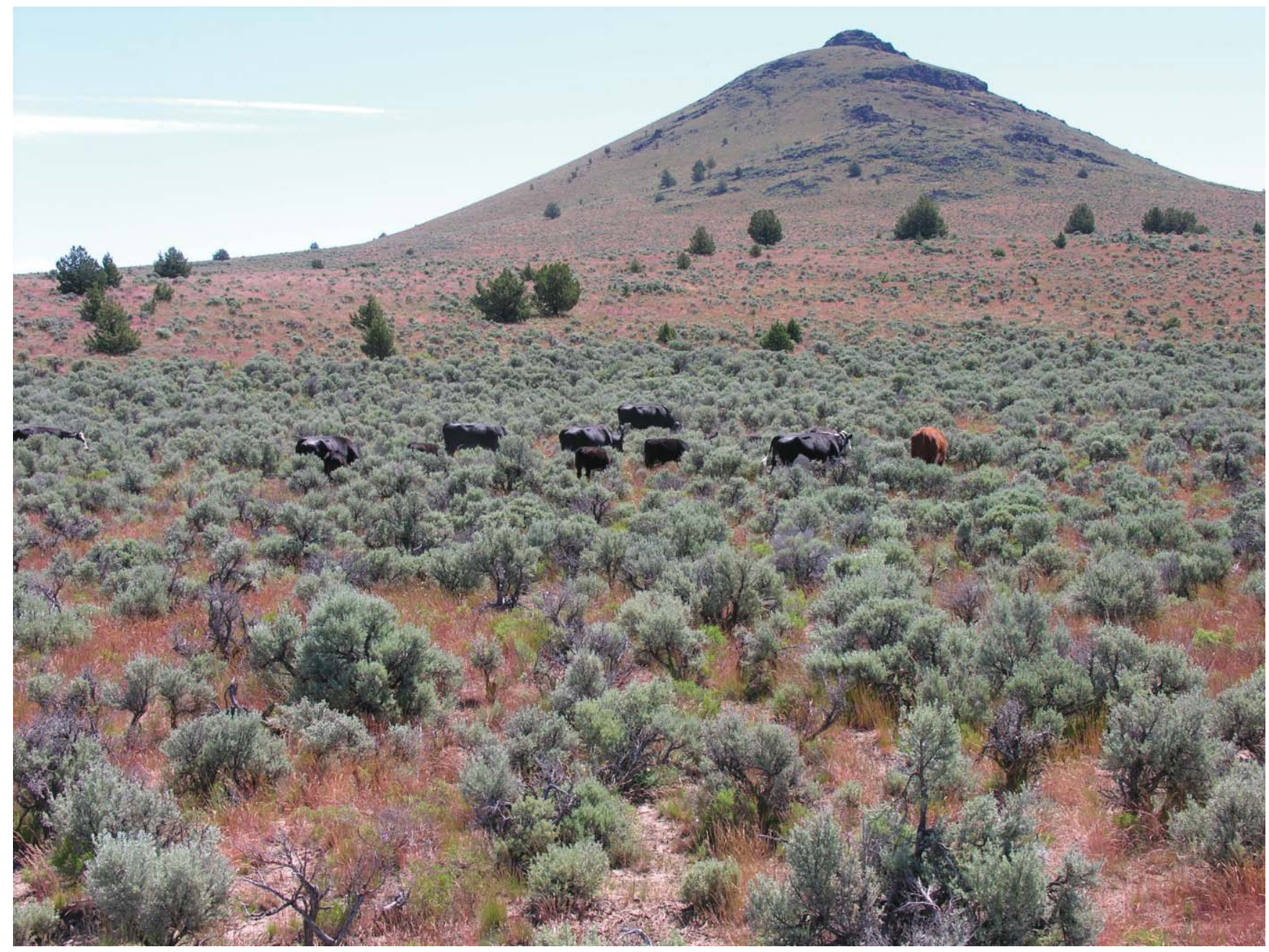

Livestock access to forage in rough or rocky terrain can be enhanced with smaller management units associated with rotational grazing systems. Photo courtesy of Tony Svejcar. 